\title{
ACCESO A LOS ALIMENTOS Y HÁBITOS ALIMENTARIOS DE JÓVENES UNIVERSITARIOS, ESTADO DE MÉXICO.
}

\author{
ACCESS TO FOOD AND EATING HABITS OF YOUNG UNIVERSITY, STATE OF MEXICO. \\ Martínez-Hernández Virginia Reyna ${ }^{1}$, Sandoval-Copado José Rogelio¹, Aranzana Juarros Miguel². \\ 1 Universidad Autónoma del Estado de México, México. 2 Universidad de León, España.
}

\begin{abstract}
RESUMEN
Introducción: Los hábitos alimentarios se adquieren en el entorno familiar y éste influye de forma importante en la elección de los alimentos. Objetivo: Analizar el acceso de alimentos y su influencia en los hábitos alimentarios de estudiantes en la Universidad Autónoma del Estado de México. Material y Método: Se encuestaron 300 estudiantes universitarios sobre consumo de alimentos, hábitos alimentarios, medidas antropométricas, y aspectos socio-culturales. Se llevaron a cabo visitas a cafeterías y negocios de comida para identificar los alimentos accesibles en el entorno de la universidad. Se realizó un análisis comparativo por sexo de las variables antropométricas mediante la prueba Z y se determinó la relación que existe entre el consumo de alimentos y los valores de IMC para peso normal y sobrepeso mediante un análisis multifactorial (MFA). Resultados: El acceso a alimentos fue muy variado, siendo más consumidos aquellos No recomendables por su relación practicidad-costo. Se observaron correlaciones positivas entre los hábitos alimentarios y los valores de IMC (sobrepeso y normal). Conclusión: El acceso a los alimentos influye en los hábitos alimentarios de los estudiantes, se observó la tendencia a elegir aquellos No recomendables por practicidad y bajo costo; se encontró una correlación positiva entre el consumo de alimentos recomendables para IMC normal y los No recomendables para sobrepeso.

Palabras Clave: Acceso a los alimentos, hábitos alimentarios, jóvenes universitarios.
\end{abstract}

\section{ABSTRACT}

Introduction: Eating habits are acquired in the family environment and this has an important influence on the choice of food. Objective: To analyze the access of food and its influence on the eating habits of students at the Universidad Autónoma del Estado de México. Material and method: 300 university students were surveyed on food consumption, eating habits, anthropometric measures, and socio-cultural aspects. Visits were made to cafeterias and food shops to identify accessible foods in the university setting. A comparative analysis by sex of the anthropometric variables was performed using the $z$ test and the relationship between food consumption and BMI values for normal weight and overweight was determined using a multifactorial analysis (MFA). Results: The access to food was very varied, being more consumed those not recommended for their practicality-cost ratio. Positive correlations were observed between dietary habits and BMI values (overweight and normal). Conclusions: Access to food influences the eating habits of students, the tendency to choose those not recommended for convenience and low cost; a positive correlation was found between the consumption of food recommended for normal body-mass index and food not recommended for overweight.

Key words: Availability of food, eating habits, university students

Correspondencia: Virginia Reyna Martínez Hernández vrmrtinezh@uaemex.mx

Recibido: 10 de abril 2021, aceptado: 17 de mayo 2021

(C) Autor2021

(c) (1)

DOI: https://doi.org/10.29105/respyn20.3-5

Citation: Martínez-Hernández V.R., Sandoval-Copado J.R., Aranzana J.M. (2021) Acceso a los alimentos y hábitos alimentarios de jóvenes universitarios, Estado de México. Revista Salud Pública y Nutrición, 20 (3), 36-45. 


\section{Introducción}

Existe un consenso en que los hábitos alimentarios se adquieren en el entorno familiar y éste influye de forma importante en la elección de los alimentos (Macias et al., 2012; Neumark-Sztainer et al., 1999; Scaglioni et al., 2008; St-Onge et al., 2003 y Young, 2004). Sin embargo, los jóvenes que inician los estudios universitarios tienen la necesidad de cambiar de residencia o desplazarse lejos del hogar con autonomía para decidir en su alimentación, con una dinámica diferente a la familiar y consumir los alimentos accesibles en el entorno escolar.

La Organización de las Naciones Unidas para la Alimentación y la Agricultura (FAO, por sus siglas en inglés), la Organización Panamericana de la Salud (OPS), el Programa Mundial de Alimentos (WFP, por sus siglas en inglés) y el Fondo de las Naciones Unidas para la Infancia (UNICEF, por sus siglas en inglés), sugieren que el acceso a los alimentos se relaciona con la forma en que las personas pueden disponer física y económicamente de los alimentos, ya sea a través de los ingresos del trabajo, producción de autoconsumo o mediante el apoyo de políticas públicas como programas de transferencias condicionadas, asistencia alimentaria y escolar (FAO, OPS, WFP y UNICEF, 2018).

De acuerdo con la Organización Mundial de la Salud (2019), los cambios en los hábitos alimentarios son consecuencia de las transformaciones ambientales y sociales por la falta de políticas de apoyo a los sectores que involucran salud, agricultura, transporte, planificación urbana y medio ambiente; debido al aumento del procesamiento, distribución y comercialización de alimentos; así como por la falta de educación sobre una alimentación adecuada por las personas.

Este panorama prevé la posibilidad de una mayor incidencia en la salud de la población asociada a problemas alimentarios que deriven en las Enfermedades Crónicas No Transmisibles (ECNT) como el síndrome metabólico que se identifica principalmente con el sobrepeso y la obesidad, (Scaglioni et al., 2008), como el cáncer, la diabetes, las enfermedades respiratorias crónicas (Gutiérrez et al., 2012). Existen otras causas que precisan de atención como son: dieta desequilibrada, inactividad física, consumo de tabaco y alcohol (OMS, 2015 y 2016). De acuerdo con los resultados de la Encuesta
Nacional de Salud y Nutrición 2018, se observa un incremento de estos padecimientos en la población adulta (>20 años), en México, por lo cual el riesgo del síndrome metabólico es alto (Campos et al., 2018).

La OMS y la Organización Panamericana de la Salud promueven una alimentación saludable mediante recomendaciones para combatir la mala alimentación (por exceso o por deficiencia), estas políticas se atienden en México por la Secretaría de Salud mediante Normas Oficiales que dictan las pautas para la orientación alimentaria (NOM-043-SSSA2-2012), especificaciones para el etiquetado de alimentos y bebidas no alcohólicas pre-envasados (NOM-051SCFI/SSA1-2010); así como para el tratamiento integral del sobrepeso y la obesidad (NOM-008SSA3-2017). Así también, el Instituto Mexicano del Seguro Social (IMSS, 2016) participa a través estrategias y programas de prevención del sobrepeso, obesidad, ECNT y la atención de las deficiencias de vitaminas y minerales.

Ante la situación de mala alimentación, el gobierno mexicano elabora Guías Alimentarias (IMSS, 2016 y Bonvechio-Arenas et al., 2015), con las recomendaciones sobre dieta y actividad física para la población en general, con el propósito de lograr una alimentación adecuada, acorde a las costumbres, los recursos socioeconómicos y los aspectos culturales, ambientales y gastronómicos.

En la guía de 2005 se proporcionan indicadores clave sobre una óptima alimentación, mediante una dieta variada, porciones de alimentos con un contenido reducido de grasa, sal y azúcar, acorde al requerimiento energético diario etario (BonvechioArenas et al., 2015), y específica los alimentos recomendables y no recomendables.

Los alimentos recomendables aportan la energía necesaria y nutrimentos que el organismo necesita, y los No recomendables se caracterizan por un alto contenido de grasa, azúcares, sal y aditivos (la energía que aportan es pasajera), por lo que son considerados como hipercalóricos. El IMSS (2016) emite información de las kilocalorías que proporcionan algunos de estos alimentos.

Los estilos de vida dependen de la interacción entre las personas y las condiciones sociales, económicas 
y ambientales que se establecen en ciertos estratos sociales, estos factores son delineados por el habitus, como la aplicación de esquemas idénticos, más allá de los límites directamente adquiridos y la necesidad inherente a las condiciones de aprendizaje; el habitus es un "sistema de disposiciones duraderas y transferibles que integra todas las experiencias pasadas, funcionando en cada momento como matriz que distribuye $y$ ordena las percepciones, las apreciaciones y las acciones de los agentes de cara a una coyuntura o acontecimiento y que ellos contribuyen a producir" (Bourdieu, 2005). Se ha mostrado como las preferencias de las personas por los alimentos se moldean dentro los estratos y prácticas basadas sobre sus propiedades nutritivas, origen y procesamiento (Atkinson y Deeming, 2015). Así, los jóvenes universitarios van conformando ciertos hábitos alimentarios con base en el capital científico, social y cultural.

En un estudio de discusión en grupos focales (141 adolescentes), se observaron factores que influyen en la elección de alimentos destacando al hambre, el antojo, el atractivo, la conveniencia del alimento y el beneficio saludable. El estudio señaló la influencia de ciertos factores ambientales como la compañía de personas, la accesibilidad, el color, olor de los alimentos, la ubicación del lugar, la iluminación, el sonido y la temperatura ambiente (Stroebele y De Castro, 2004).

Otro estudio, en los Estados Unidos de Norteamérica, se encontró una disponibilidad mayor de alimentos menos sanos en las escuelas (Delva et al., 2007), además una asociación lineal entre el nivel socioeconómico, grado académico de los padres y la elección de alimentos más sanos. Se sugirió que las escuelas deberían disminuir la disponibilidad de estos alimentos y evaluar el papel de la industria de alimentos en la solución del problema, en concordancia con las sugerencias de la OMS (2019). En México, la investigación sobre la alimentación en el sector estudiantil universitario es escasa, aunque se observa en las cafeterías universitarias el acceso amplio y variado de alimentos, y el predominio de la oferta de los No recomendables como antojitos (alimentos típicos de la región). Este sector de jóvenes conforma un estrato con el capital científico, social y cultural base para tomar decisiones y un papel más activo en el logro de una alimentación sana y la prevención de las ECNT.
El objetivo del presente trabajo consistió en analizar el acceso de alimentos en el entorno universitario y su influencia en los hábitos alimentarios de alumnos en la Universidad Autónoma del Estado de México (UAEMéx).

\section{Material y Método}

Se llevó a cabo una investigación transversal y descriptiva, con estudiantes de la Universidad Autónoma del Estado de México, mediante una muestra al azar de diversas licenciaturas, 150 hombres y 150 mujeres, con edades entre 18 y 23 años (media=20 y desviación estándar $=1.40$ ), en la ciudad de Toluca, se excluyeron a estudiantes de la licenciatura de nutrición, mujeres embarazadas, y personas con ablación. Se aplicó un cuestionario sobre hábitos alimentarios, dirigida por alumnos de la licenciatura de Químico en Alimentos (LQA), se consideraron medidas antropométricas, variables socio-culturales, así como la frecuencia y lugar de consumo de alimentos recomendables y No recomendables. La participación fue libre y se firmó la carta de consentimiento para el uso de la información.

El cuestionario fue diseñado por expertos multidisciplinarios, contestado en una prueba piloto por 25 estudiantes de la LQA, se evaluó la claridad de las preguntas y el tiempo de respuesta; en 2018 , fue aplicado en forma dirigida por cinco estudiantes, previamente entrenados.

Variables de análisis

Antropométricas: sexo (mujer u hombre); edad (años cumplidos); peso (kilogramos); y estatura (centímetros). Consumo de alimentos: desayuno (sí o no); refrigerio (sí, no). Número de comidas diarias $(1,2,3,>3)$. Tipo y frecuencia del consumo de alimentos Recomendables (comida completa "corrida", jugos naturales, agua, frutas y verduras); y No recomendables (tortas, sopes, tlacoyos, quesadillas, tamales, atole, tacos de guisado fritos, refrescos, jugos procesados, frituras y cerveza).

Socio-culturales: padres: grado de estudios (sin estudios, primaria, secundaria, preparatoria/técnica, licenciatura, posgrado) y ocupación (trabaja, estudia, ama(o) de casa, retirada(o), ausente). Con quién vivían (padres, familiares, estudiantes, solo). Lugar acostumbrado para comer (cafetería, puestos, 
cocinas económicas, lleva comida preparada en casa).

Se obtuvo el índice de Masa Corporal (indicador que relaciona el peso y la talla, utilizado para identificar sobrepeso y obesidad en los adultos), mediante los datos declarados por los estudiantes y la fórmula (peso en kilogramos/talla en metros elevada al cuadrado), y clasificado como: Bajo peso<18.5, Normal 18.5-24.9, Sobrepeso 25.0-29.9, Obesidad I 30.0-34.9, Obesidad Extrema II 35.0-39.9, Obesidad Extrema III > 40, de acuerdo con el apéndice normativo C de la NOM-043-SSA2-2012.

Para el análisis de la información se aplicó la prueba chi-cuadrada que determinó la normalidad de las distribuciones por sexo (talla, peso e IMC); se obtuvo la estadística descriptiva (media y desviación estándar) de variables antropométricas y socioculturales; el análisis comparativo para la diferencia de porcentajes de sexo para IMC (normal y sobrepeso) mediante la prueba z. Se obtuvieron las gráficas de componentes principales mediante análisis multifactorial (MFA) para consumo y lugar de acceso a los alimentos e IMC, con Rstudio (IDE, versión 3.5.1).

Se llevaron a cabo visitas de campo a cafeterías y negocios de comida para identificar mediante un sondeo los alimentos recomendables y No recomendables, accesibles en el entorno de la universidad, ubicados en un tiempo no mayor a 15 minutos a pie, en Ciudad Universitaria, Colón, Los Uribe (zona urbana) y El Cerrillo (zona rural).

\section{Resultados}

Se exponen en el orden: 1. El acceso de alimentos en la UAEMéx, 2. Los hábitos alimentarios de los estudiantes y 3. Relación de alimentos y lugar de consumo por los estudiantes de IMC normal y sobrepeso.

1. El acceso de alimentos en la UAEMéx Los estudiantes consumen alimentos que se expenden en las cafeterías ubicadas en la universidad $(53 \%)$ y en negocios aledaños (8\%), y consumen refrigerios elaborados en casa $(83 \%)$, durante las actividades académicas.

Con base en los alimentos disponibles en las cafeterías universitarias y negocios aledaños, los estudiantes consideraron que su alimentación fue regular (64\%), adecuada (16\%) e inadecuada (20\%); y su forma de alimentación les permitió un desempeño académico adecuado (45\%), regular $(46.7 \%)$ e inadecuado $(9 \%)$.

\section{Cafeterías en la universidad}

Las cafeterías mostraron una variedad de alimentos típicos que abarcaban antojitos (sopes, enchiladas, quesadillas) tacos fritos, tortas, comida rápida (pizza, hot dogs), ensaladas, desayunos y platillos más elaborados con pescado y carne. Todas tenían una sección de productos procesados (botanas, galletas, dulces, refrescos, jugos) en aparadores; fuera y dentro había máquinas expendedoras de galletas, botanas y café; en algunas por fuera había puestos de tacos fritos, gorditas, pambazos, tortas, quesadillas, ensaladas, baguetes; helados y dulces.

Los estudiantes podían comprar un alimento en el rango de $\$ 16$ a $\$ 50$ pesos mexicanos. Las comidas completas estaban en el intervalo de $\$ 33$ a $\$ 45$, considerándose accesibles con base en un salario mínimo (\$123 MXN).

Seis de las 21 cafeterías visitadas $(28.5 \%)$ ofrecían precios más económicos, y sólo tres, raciones más pequeñas de alimentos y limpieza adecuada en el servicio.

Negocios aledaños a la universidad

Se encontró una gran variedad de alimentos como pizza, hot dogs, tortas, jugos naturales, licuados, tamales, atole, pastes, comida corrida, antojitos, tacos de carnitas y de canasta, quesadillas fritas y al comal, tacos fritos (carne y guisados), dulces, helados y papas fritas.

Aunque la mayoría de los alimentos disponibles en el entorno universitario son del mismo tipo, en la zona rural se ofrecen más tacos fritos y en la zona urbana, más comida rápida. Los alimentos hipercalóricos fueron más económicos y de acceso para el consumo diario.

2. Los hábitos alimentarios de los estudiantes Dado que el horario de clases de los estudiantes que fue mixto $(58.3 \%)$, matutino $(28 \%)$, y vespertino $(11.7 \%)$, se observa que ellos requieren del consumo de alimentos accesibles en el entorno universitario. 


\section{Artículo Original}

Alimentos principales: desayuno, comida, cena y refrigerio

Sobre el consumo de los alimentos principales, aquellos que realizaron tres comidas al día fueron $(50.7 \%)$, dos comidas, $(27.3 \%)$, una comida $(3 \%)$, más de tres comidas mediante refrigerios (19\%). El $50 \%$ de alumnos omitieron alguna de las tres comidas principales del día (desayuno, comida o cena), presentando ayunos durante el día en su estancia en la universidad.

El consumo de estos alimentos principales se lleva a cabo en forma diferenciada, la tercera parte omite el desayuno $(27.6 \%)$ y la cena $(13.3 \%)$, y la mayoría (99.3) realiza la comida de medio día (tabla 1).

Tabla 1. Consumo de los alimentos principales del día por los estudiantes

\begin{tabular}{lrl}
\hline $\begin{array}{l}\text { Alimentos } \\
\text { principales }\end{array}$ & $\begin{array}{r}\text { Frecuencia de } \\
\text { consumo }\end{array}$ & $\%$ \\
\hline Desayuno & 217 & 72.3 \\
Comida de & 298 & 99.3 \\
medio dia & 210 & 86.7 \\
Cena & 248 & 82.7 \\
Refrigerio &
\end{tabular}

Fuente: Base de datos propia.

La comida de medio día la realizaron en las cafeterías universitarias $(52.7 \%)$, en los negocios aledaños $(8 \%)$, comieron en casa $(21.7 \%)$ y prepararon sus alimentos y llevaron lunch $(17 \%)$.

Cenaron en casa $(83.3 \%)$, en puestos $(2.7 \%)$, en la cafetería universitaria $(0.7 \%)$; y aquellos que cenaron en puestos y cafeterías, por lo general, vivían solos y consumían más alimentos hipercalóricos.

El consumo de alimentos por los estudiantes en mayor medida son los No recomendables, (figura 1).
Figura 1. Porcentaje de consumo de alimentos recomendables y No recomendables por los estudiantes de la UAEMéx, 2018.

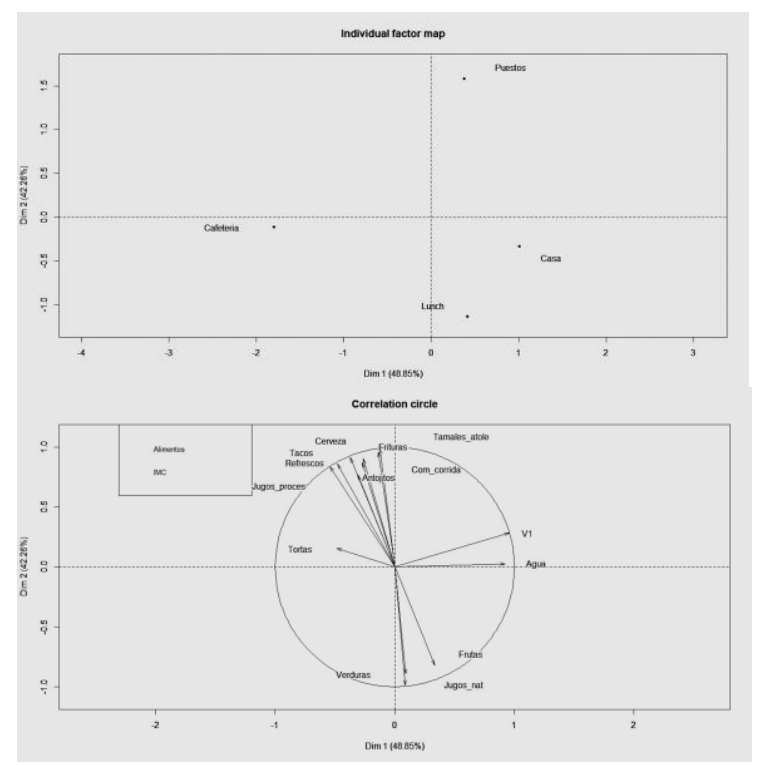

Aspectos socio-culturales

El grado de estudios de las madres de familia fue: posgrado (5.30\%), licenciatura (38.7\%) y preparatoria $(25.7 \%)$; y los padres: posgrado $(7.3 \%)$ licenciatura $(45.3 \%)$ y preparatoria (19.7\%). La mayoría de los padres $(89.3 \%)$ y madres $(55.3 \%)$ trabajan; los estudiantes cuentan con el capital social y la solvencia económica para observar una mejor salud.

Las madres de los estudiantes fueron amas de casa $(40 \%)$, y dado que más de la mitad de ellos tiene un horario mixto en la escuela, logran la autonomía de la selección de sus alimentos.

Medidas antropométricas

Se encontró que las mujeres tuvieron un peso y estatura menor que los hombres (tabla 2). Las distribuciones no fueron normales de acuerdo a chicuadrada y alfa $=0.05$. 
Tabla 2. Peso, estatura e IMC, hombres y mujeres

\begin{tabular}{clrr}
\hline \multicolumn{2}{c}{ Sexo } & Hombres & Mujeres \\
\hline \multirow{2}{*}{ Peso } & Media & 70.9 & 58.4 \\
& Desv. típ. & 10.3 & 8.2 \\
\multirow{3}{*}{ Estatura } & Media & 1.7 & 1.6 \\
& Desv.tip & 0.1 & 0.1 \\
& Media & 23.9 & 22.8 \\
IMC & Media & 3.1 & 2.9 \\
& Desv. tip. & \\
\hline
\end{tabular}

Fuente: Base de datos propia.

Los estudiantes tuvieron un IMC normal (70.3\%), con sobrepeso (25\%), obesidad tipo I (2,3\%), y tipo II $(0.3 \%)$, de esta manera más de una cuarta parte presentó problemas relacionados con el síndrome metabólico (tabla 3).

Tabla 3. Porcentajes de IMC para hombres y mujeres.

\begin{tabular}{lrrrrrr}
\hline \multicolumn{1}{c}{ IMC } & \multicolumn{2}{c}{ Hombres } & \multicolumn{2}{c}{ Mujeres } & \multicolumn{2}{c}{ Total } \\
& No. & \multicolumn{1}{c}{$\%$} & No. & $\%$ & No. & $\%$ \\
\hline Bajo peso & 2 & 1.3 & 4 & 2.7 & 6 & 2 \\
Normal & 97 & 64.7 & 114 & 76 & 211 & 70.3 \\
Sobrepeso & 45 & 30 & 30 & 20 & 75 & 25 \\
Obesidad I & 6 & 4 & 1 & 0.7 & 7 & 2.3 \\
Obesidad II & 0 & -- & 1 & 0.7 & 1 & 0.3 \\
Total & 150 & 100 & 150 & 100 & 300 & 100 \\
\hline
\end{tabular}

Fuente: Base de datos propia.

Se encontraron diferencias entre los porcentajes de IMC: normal y sobre peso, entre hombres y mujeres, los hombres presentaron un porcentaje más alto, con base en $\mathrm{z}=2.00$ y $\mathrm{p}=0.062$, (tabla 4 ); estos índices fueron menores a los reportados por la población de acuerdo con ENSANUT, 2018 (Campos et al., 2018).
Tabla 4. Resultados en diferencia de porcentajes entre hombres y mujeres. IMC normal, sobre peso, obesidad I

\begin{tabular}{lrrrr}
\hline \multirow{2}{*}{ IMC } & \multicolumn{2}{c}{ Hombres } & \multicolumn{2}{c}{ Mujeres } \\
& No. & \multicolumn{1}{c}{$\%$} & \multicolumn{1}{c}{ No. } & \multicolumn{1}{c}{$\%$} \\
\hline Normal & 97 & 65.5 & 114 & 78.6 \\
Sobrepeso* $^{*}$ & 45 & 30.4 & 30 & 20.7 \\
Obesidad I $^{*}$ & 6 & 4.1 & 1 & 0.7 \\
Total & $148^{*}$ & 100.0 & 145 & 100.0 \\
\hline
\end{tabular}

Fuente: Base de datos propia. ${ }^{*} \mathrm{p}<.05$

3. Relación de alimentos y lugar de consumo por los estudiantes de IMC normal

El MFA explica el $91.1 \%$ de la varianza. Se observó para peso normal el consumo de alimentos recomendables (agua, verduras, frutas y jugos naturales) en casa y como lunch (refrigerio); mientras, en la cafetería, consumen tortas, antojitos y comida corrida, y en los puestos, tamales, atole, jugos procesados, tacos y cerveza. Los valores de IMC en el rango de peso normal tienen una correlación en orden decreciente con el consumo de alimentos en casa, lunch, puestos y cafetería. La dimensión uno discrimina entre los lugares de consumo y la dimensión 2 entre alimentos recomendables y No recomendables (figura 2).

Figura 2. Análisis de componentes principales (MFA) para los alimentos consumidos en diferentes lugares y su relación con peso normal.

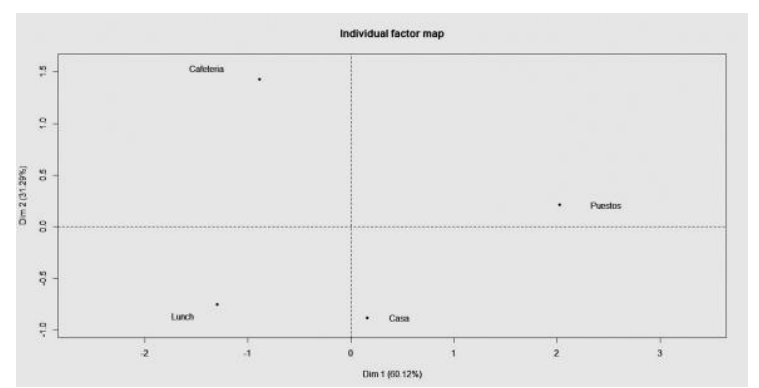




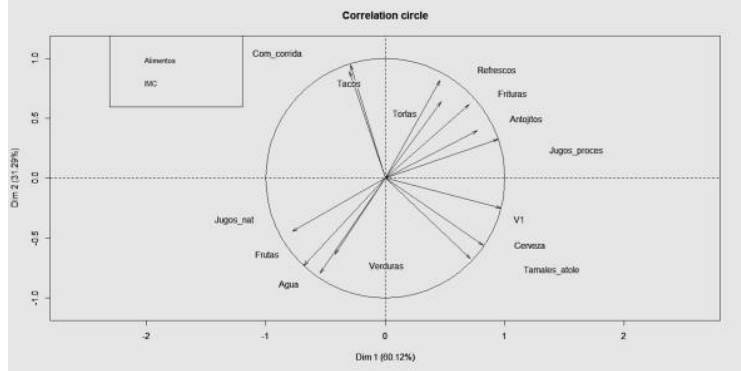

Relación de alimentos y lugares de consumo para los estudiantes con sobrepeso

El MFA explica el $91.4 \%$ de la varianza. Se observó para sobrepeso el consumo de alimentos recomendables (agua, verduras, frutas y jugos naturales) como lunch y en menor grado en casa. Se favorece la alimentación en puestos consumiendo antojitos, jugos procesados, refrescos, frituras, tamales, atole y tortas. En la cafetería, consumen principalmente comida corrida y tacos. El sobrepeso tiene una correlación en orden decreciente con el consumo de alimentos en puestos, no consumo en casa, lunch, y cafetería. La dimensión uno discrimina entre alimentos recomendables y No recomendables y la dimensión 2 entre lugares de consumo (figura 3).

Figura 3. Análisis de componentes principales (MFA) para los alimentos consumidos en diferentes lugares y su relación con sobrepeso.

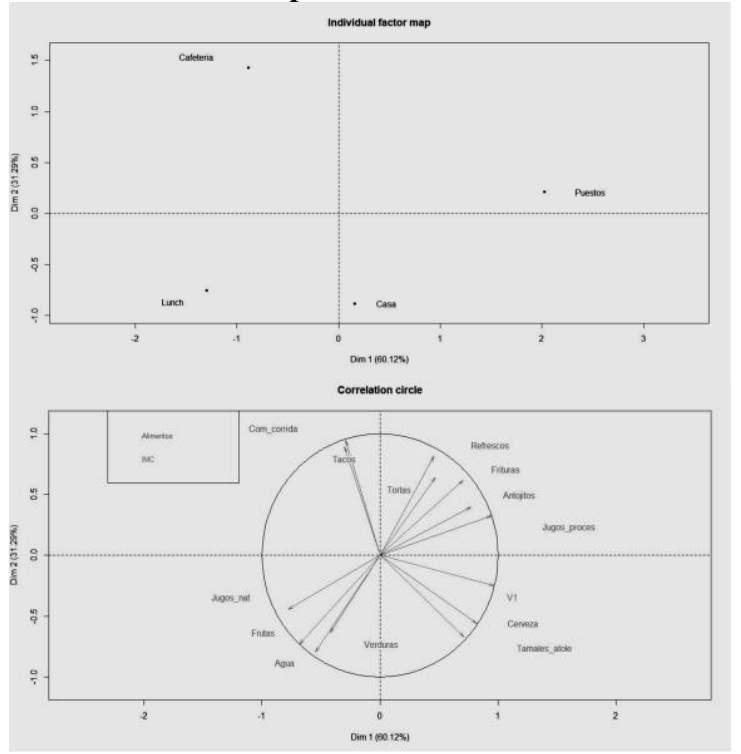

\section{Discusión}

A partir del análisis del acceso a los alimentos en el entorno universitario, se observó la disponibilidad de una gran variedad éstos, y la selección principalmente de los No recomendables por los estudiantes con peso normal y sobrepeso, esto por practicidad, y quizá por considerar prioritaria la asistencia a clases y el cumplimiento de actividades escolares como pueden ser tareas, trabajos en equipo y el servicio social, lo cual los lleva a prolongar su estancia en la escuela; e influye el costo más económico de éstos alimentos; se adiciona el hecho de que un porcentaje importante de las madres de los estudiantes trabajan, por lo cual muchos de ellos han adquirido la autonomía sobre su alimentación que se observa pormenorizada.

Se encontró que los hábitos alimentarios para muchos de los estudiantes no son los adecuados, dado que una tercera parte omitieron una de las tres comidas principales del día y otros sustituyeron las comidas por alimentos No recomendables, provocando un desequilibrio energético por ayuno o falta de nutrientes y en consecuencia la presencia de posibles "atracones" que resultan en un mayor consumo de alimentos para satisfacer el hambre, y la ingesta de un alto aporte calórico en el momento de disponer de éstos, lo cual es un antecedente del síndrome metabólico y las ECNT. En trabajos reportados sobre el ayuno (IMSS, 2016 y MartínezHernández, 2014), nutrición y neurofisiología de la alimentación (Navarro-Meza et al., 2016) se han observado efectos de la supresión de alimentos no controlada en el organismo, como las alteraciones de los procesos de la ingesta de los nutrimientos, la falta de la dopamina en el cerebro y de trastornos alimenticios; el buen funcionamiento del organismo requiere de los nutrimentos equilibrados, variados y acorde al gasto energético que las personas realizan.

En esta situación, los estudiantes pueden presentar problemas de concentración, rendimiento escolar y malestares como gastritis, dolor de cabeza y colitis si la ingesta de alimentos no es adecuada, ya que las actividades académicas demandan un alto aporte energético que solo se logra con una dieta balanceada que cumpla con las recomendaciones establecidas en las guías alimentarias y de la actividad física (IMSS, 2016 y Bonvechio-Arenas et al., 2015). 
A propósito de los aportes nutrimentales de los alimentos, se observó que una gran cantidad de alimentos típicos se expenden a granel tanto en cafeterías y negocios aledaños, pero se desconoce si éstos se elaboran con apego a las especificaciones de nutrimentos indicados en las guías alimentarias desarrolladas para la población mexicana, por lo que se propone realizar estudios futuros sobre la evaluación de la calidad nutrimental de los alimentos accesibles en el entorno universitario.

Se encontró la incidencia de mayor porcentaje de sobrepeso en hombres, que no ha sido reportada en el sector de jóvenes y pude resultar un tema de interés en futuras investigaciones.

Los estudiantes universitarios cuentan con el capital científico, ya que han sido involucrados en temas de alimentación saludable en sus estudios antecedentes, disponen de información sobre la forma de alimentarse, y su entorno familiar les proporciona el capital social y cultural, dado que la mayoría vive en familia, los padres cuentan con estudios superiores, y la mayoría de ellos trabajaba, así se puede suponer cierta solvencia económica para el acceso a alimentos de calidad (nutritivos, frescos, limpios), lo cual permitiría a este sector lograr una alimentación adecuada y prevenir la incidencia de las ECNT asociadas al sobrepeso y obesidad.

En concordancia con Bourdieu (2005), la dinámica del entorno universitario se constituye como el espacio social, que con el tiempo va moldeando los gustos y preferencias alimentarias de los estudiantes, desarrollado cierto habitus alimentario, favorecido por la interacción de los compañeros que se convierte en prácticas cotidianas, favorecidas por el inadecuado acceso a los alimentos y la falta de atención a los hábitos alimentarios.

\section{Conclusiones}

Se encontró que el acceso a los alimentos influye en los hábitos alimentarios de los estudiantes que participaron, observándose una tendencia a elegir los alimentos No recomendables debido a que por su practicidad y bajo costo representan una opción adecuada para su acelerado estilo de vida. Estos hábitos alimentarios pueden tener un impacto en la incidencia y prevalencia de sobrepeso y obesidad entre los jóvenes universitarios, ya que se encontró una correlación positiva entre el consumo de alimentos recomendables con los valores del IMC para peso normal y de alimentos No recomendables con sobrepeso.

Las autoridades universitarias deberían de promover la educación de hábitos saludables en los jóvenes para prevenir las ECNT en este sector, mediante la introducción de contenidos sobre la alimentación adecuada, el establecimiento de los horarios de clases considerando los horarios de comida; así como estrategias sobre el acceso de alimentos de calidad en el entorno universitario, aprovechando el capital social, cultural y científico que conforma el campo de la alimentación en el entorno universitario.

Se sugiere continuar la realización de estudios longitudinales y sectoriales para conocer la incidencia de los problemas de síndrome metabólico y las ECNT.

\section{Agradecimientos}

Al Dr. Javier González Gallego, Departamento de Biomedicina. Universidad de León, España, por las facilidades otorgadas para el acceso a los recursos electrónicos considerados en la redacción del artículo, durante la estancia de investigación realizada.

\section{Bibliografía}

Atkinson, W., \& Deeming, C. (2015). Class and Cuisine in Contemporary Britain: the Social Space, the Space of Food and Their Homology. The Sociological Review, 63(4), 876-896. doi: 10.1111/1467-954x.12335

Bonvechio-Arenas, A., Fernández-Gaxiola A.C., Plaza B.M.; Kaufer-Horwitz, M.; Pérez, L.A.B.; Rivera, D.J.Á. (2015) Guías alimentarias y de actividad física en contexto de sobrepeso y obesidad en la población mexicana. México: Academia Nacional de Medicina. Recuperado de: https://www.insp.mx/resources/images/stories/2 015/Noticias/Nutricion_y_Salud/Docs/151118_g uias_alimentarias.pdf

Bourdieu, P. (2005) Una Invitación a la sociología reflexiva. Argentina: Siglo XXI.

Dávila-Torres, J., González-Izquierdo, J., \& BarreraCruz, A. (2015). Panorama de la obesidad en 
México. Revista Médica del Instituto Mexicano del Seguro Social, 53(2), 240-249. Recuperado de

http://revistamedica.imss.gob.mx/editorial/index .php/revista_medica/article/view/21/72

Delva, J., O’Malley, P. M., \& Johnston, L. D. (2007). Availability of More-Healthy and Less-Healthy Food Choices in American Schools. American Journal of Preventive Medicine, 33(4), S226S239. doi: 10.1016/j.amepre.2007.07.018

Campos, N. I., Hernández B. L., Espinosa J., Padilla F. D., Barquera, S. Estado de nutrición y tensión arterial en adultos en Encuesta Nacional de Salud y Nutrición (ENSANUT). (2018). Informe final. La Secretaría de Salud, el Instituto Nacional de Salud Pública y el Instituto Nacional de Estadística y Geografía. México. Recuperado de:

https://ensanut.insp.mx/encuestas/ensanut2018/d octos/informes/ensanut_2018_presentacion_resu ltados.pdf

FAO, OPS, WFP y UNICEF (2018). Panorama de la seguridad alimentaria y nutricional en América Latina y el Caribe. Santiago. Recuperado de: https://iris.paho.org/bitstream/handle/10665.2/49 616/9789251310595_spa.pdf

Instituto Mexicano del Seguro Social, IMSS (2016). Nutrición. Promoción de la Salud. Recuperado de:

http://www.imss.gob.mx/sites/all/statics/salud/g uias_salud/hombres/Guia_hombres_nutricion.pd $\mathrm{f}$

Macias M. A. I., Gordillo S. L. G., \& Camacho R. E. J. (2012). Hábitos alimentarios de niños en edad escolar y el papel de la educación para la salud. Revista chilena de nutrición, 39(3), 40-43. doi: 10.4067/S0717-75182012000300006

Martínez-Hernández A. (2014) Nutrición saludable frente a la Obesidad Bases científicas y aspectos dietéticos. México: Editorial Médica Panamericana.

Navarro-Meza M, García C. K. A.; Mendoza del Toro, P.G. y Miramontes S. M. (2016). Neurofisiología de la Alimentación en La
Educación en Alimentación y la Nutrición. México: McGraw Hill Educación.

Neumark-Sztainer, D., Story, M., Perry, C., \& Casey, M. A. (1999). Factors Influencing Food Choices of Adolescents. Journal of the American Dietetic Association, 99(8), 929-937. doi: 10.1016/s0002-8223(99)00222-9

Organización Mundial de la Salud OMS. (2019). Nota descriptiva Enfermedades cardiovasculares. Centro de prensa, Recuperado de:

http://www.who.int/mediacentre/factsheets/fs31 7/es/

Organización Mundial de la Salud (2015). Nota Descriptiva Enfermedades cardiovasculares. Centro de prensa. Recuperado de: http://www.who.int/mediacentre/factsheets/fs31 7/es/.

Organización Mundial de la Salud. (2016). Nota descriptiva No. 311 Obesidad y sobrepeso. Centro de prensa, (OMS). Recuperado de: http://www.who.int/mediacentre/factsheets/fs31 $1 / \mathrm{es} /$.

Scaglioni, S., Salvioni, M., \& Galimberti, C. (2008). Influence of parental attitudes in the development of children eating behavior. British Journal of Nutrition, $\quad 99(\mathrm{~S} 1), \quad$ S22-S25. doi: $10.1017 / \mathrm{s} 0007114508892471$

Secretaria de Salud (México) Norma Oficial Mexicana NOM-043-SSA2-2012, Servicios básicos de salud. Promoción y educación para la salud en materia alimentaria. Criterios para brindar orientación. Apéndice normativo $C$. Diario Oficial de la Federación. Recuperado de: http://www.salud.gob.mx/unidades/cdi/nom/com pi/043ssa205.pdf

Secretaría de Economía (México) Norma Oficial Mexicana. NOM-051-SCFI/SSA1-2010. Especificaciones generales de etiquetado para alimentos $y$ bebidas no alcohólicas preenvasados-Información comercial y sanitaria. Diario Oficial de la Federación. Recuperado 
http://www.dof.gob.mx/normasOficiales/4010/s eeco11_C/seeco11_C.htm

Secretaría de Salud (México). Norma Oficial Mexicana. NOM-008-SSA3-2017. Para el tratamiento integral del sobrepeso y la obesidad. Diario Oficial de la Federación. Recuperado de: http://www.dof.gob.mx/nota_detalle.php?codigo $=5523105 \&$ fecha $=18 / 05 / 2018$

Stroebele, N., \& De Castro, J. M. (2004). Effect of ambience on food intake and food choice. Nutrition, 20(9), 821-838. doi: 10.1016/j.nut.2004.05.012

St-Onge, M. P., Keller, K. L., \& Heymsfield, S. B. (2003). Changes in childhood food consumption patterns: a cause for concern in light of increasing body weights. The American Journal of Clinical Nutrition, 78(6), 1068-1073. doi:10.1093/ajen/78.6.1068

Young, E. M., Fors, S. W., \& Hayes, D. M. (2004). Associations between Perceived Parent Behaviors and Middle School Student Fruit and Vegetable Consumption. Journal of Nutrition Education and Behavior,36(1), 2-12. doi: 10.1016/s1499-4046(06)60122-X 